\title{
STATE-DEPENDENT DELAYED SWEEPING PROCESS WITH A NONCOMPACT PERTURBATION IN BANACH SPACES
}

\author{
M.S. Abdo, A.G. Ibrahim, S.K. Panchal
}

Abstract. This paper is concerned with the existence of solutions for a state dependent time delayed sweeping processes with a noncompact nonconvex perturbation in $p$-uniformly convex and $q$-uniformly smooth Banach.

\section{Mathematics Subject Classification: 34A60.}

Keywords: sweeping process, normal cone, duality mapping, $p$ - uniformly convex and $q$-uniformly smooth Banach spaces, generalized projection.

\section{INTRODUCTION}

In his leading paper, Moreau [19] proposed and studied the following differential inclusion governed by sweeping process of first order:

$$
\left\{\begin{array}{l}
-u^{\prime}(t) \in N_{C(t)}(u(t)) \text { a.e. on } I=[0, T], \\
u(0)=u_{0},
\end{array}\right.
$$

where $C: I \rightarrow H$ is a set-valued map with nonempty closed convex subsets of a Hilbert space $H$ and $N_{C(t)}(u(t))$ is the normal cone of the subset $C(t)$ at the point $u(t)$. The problem (1) corresponds to several important mechanical problems [19].

Since then, important improvements have been developed by weaken assumptions in order to obtain the most general result of existence for sweeping processes (see for example $[5,9,10,16,22,23]$ ) in Hilbert spaces setting and $[2,7,8,18]$ in Banach spaces setting. We observe that all these papers except $[2,5]$ were without perturbation (like [7]) or with compact valued perturbation.

In [5], Aitalioubrahim proved the existence of solutions for the following delayed sweeping process:

$$
\left\{\begin{array}{l}
u(t)=\varphi(t), \text { for } t \in[-r, 0] \\
u(t)=\varphi(0)+\int_{0}^{t} u^{\prime}(s) d s, \text { for } t \in I \\
u(t) \in C(t), \text { for } t \in I \\
u^{\prime}(t) \in-N_{C(t)}(u(t))+F(t, \tau(t) u), \text { a.e. for } t \in I,
\end{array}\right.
$$


M.S. Abdo, A.G. Ibrahim, S.K. Panchal - Sweeping Process in Banach Spaces

where $C: I \rightarrow H$ is a set-valued map with nonempty compact values of a Hilbert space $H, r$ is a positive real number, $\mathcal{C}_{r}=\mathcal{C}([-r, 0], H)$ is the Banach space of continuous functions from $[-r, 0]$ to $H$ endowed with the uniform norm $\|u\|_{\infty}=$ $\sup \{\|u(t)\|: t \in[-r, 0]\}$ and $F: I \times \mathcal{C}_{r} \rightarrow H$ is a set-valued map with nonconvex noncompact values. This result is very interested because the values of the perturbation $F$ are not necessarily compact.

AL-Adsani and Ibrahim in [2], extended Aitalioubrahim's result in [5] to infinite dimensional Banach spaces and considered the following sweeping process:

$$
\left\{\begin{array}{l}
u(t)=J(\varphi(t)), w(t)=J(\psi(t)) \text { for } t \in[-r, 0] \\
u(t)=J(\varphi(0))+\int_{0}^{t} u^{\prime}(s) d s, \text { for } t \in I=[0, T] \\
J^{*}(u(t)) \in C(t, w(t)), \text { for } t \in I \\
w(t)=\psi(0)+\int_{0}^{t} J^{*} u(s) d s, \text { for } t \in I \\
u^{\prime}(t) \in-N_{C(t, w(t))}\left(J^{*} u(t)\right)+F\left(t, \tau(t) w, \tau(t) J^{*} u\right), \text { a.e. for } t \in I,
\end{array}\right.
$$

where $C: I \times X \rightarrow X$ is a set-valued map with nonempty closed convex values of Banach space $X$ and $F: I \times \mathcal{C}_{r} \times \mathcal{C}_{r} \rightarrow X^{*}$ is a set-valued map with nonconvex noncompact values in the dual space $X^{*}$ of $X$.

In [17], Ibrahim and AL-Adsani, considered the following sweeping process with noncompact valued perturbation and with delay

$$
\left\{\begin{array}{l}
u(t)=\psi(t) \text { for } t \in[-r, 0] \\
u(t)=\psi(0)+\int_{0}^{t} u^{\prime}(s) d s, \text { for } t \in I=[0, T] \\
u(t) \in C(t), \text { for } t \in I \\
u^{*}(t)=J(u(t)), \text { for } t \in I \\
\left(u^{*}\right)^{\prime}(t) \in-N_{C(t)}(u(t)+F(t, \tau(t) u), \text { a.e. for } t \in I,
\end{array}\right.
$$

where $C$ a set-valued map from $I$ to the family of nonempty closed convex subsets of $E$, and $F: I \times \mathcal{C}_{r} \rightarrow E^{*}$ is a set-valued map with nonempty closed values in the dual space $E^{*}$ of $E$. They also overcame the arising problem from the nonlinearity of the normalized mappings by based on Hausdorff measure of noncompactness.

Motivated by the above studies, and inspired by [5] and [17], in this paper, we give an existence result for another sweeping process with a noncompact perturbation in Banach spaces. Indeed, we find the sufficient conditions that guarantee the existence of two continuous functions $u:[-r, T] \rightarrow X$ and $w:[-r, T] \rightarrow X, I=[0, T](T>0)$ such that $u$ and $w$ are absolutely continuous functions on $I$ and that the following 
delayed perturbed sweeping process is satisfied

$$
\left\{\begin{array}{l}
u(t)=\varphi(t), w(t)=\psi(t), \text { for } t \in[-r, 0] \\
u(t)=\varphi(0)+\int_{0}^{t} u^{\prime}(s) d s, \text { for } t \in I \\
u^{\prime}(t) \in C(t, w(t)), \text { for a.e. } t \in I \\
w(t)=\psi(0)+\int_{0}^{t} u(s) d s, \text { for } t \in I \\
J\left(u^{\prime}(t)\right) \in-N_{C(t, w(t))}\left(u^{\prime}(t)\right)+G(t, \tau(t) w, \tau(t) u), \text { a.e. for } t \in I
\end{array}\right.
$$

where $C: I \times X \rightarrow X$ is a set-valued map with nonempty closed convex values and $G: I \times \mathcal{C}_{r} \times \mathcal{C}_{r} \rightarrow X^{*}$ is a set-valued map with nonconvex noncompact values, $\varphi, \psi \in \mathcal{C}_{r}$ are given with $\varphi(0) \in C(0, \psi(0)), J$ is the normalized duality map in $X$ and for each $t \in[0, T], \tau(t): \mathcal{C}_{X}([-r, T]) \longrightarrow \mathcal{C}_{r},(\tau(t) f)(s)=f(s+t), \forall s \in$ $[-r, 0]$.

In order to explain the mathematical motivation for this work, we mention some recent results in this domain. Castaing et al. [11] considered a second order sweeping process without delay in a separable Hilbert space $H$ in the case when $C$ is a Lipschitz set-valued map defined on $I \times H$, and taking a closed $\rho$-prox-regular $(\rho>0)$ values in $H$ and $G$ is a convex weakly compact valued scalary u.s.c defined on $I \times H \times H$ and satisfying the growth condition

$$
G(t, x, y) \subseteq(1+\|x\|+\|y\|) \mathbb{B}_{H},
$$

for all $(t, x, y) \in I \times H \times H$. Bounkhel et al.[8] considered various sweeping process with compact valued perturbations in Banach spaces and the values of the perturbations are contained in a fixed convex compact subset of $X^{*}$.

The fundamental importance in the present paper is that the values of the perturbations are neither compact nor convex and that the space is infinite dimensional Banach space. Moreover our technique allows to consider many sweeping process with noncompact perturbations in Banach spaces.

The paper is organized as follows. Section 2 is devoted to some definitions and notations needed later. In section 3 , we prove the main result (Theorem 8).

\section{Preliminaries AND NOtations}

In the following, we present some basic definitions, and preliminary facts which are used throughout this paper. Let $I=[0, T](T>0)$, and $X$ be a Banach space with topological dual space $X^{*}$. Let $\mathbb{B}=\{x \in X:\|x\| \leq 1\}$ and $\mathbb{B}^{*}=\{z \in$ $\left.X^{*}:\|z\| \leq 1\right\}$. For $x \in X$ and for nonempty subsets $A, B$ of $X$ we denote $d(x, A)$ 
M.S. Abdo, A.G. Ibrahim, S.K. Panchal - Sweeping Process in Banach Spaces

the real $\inf \{\|y-x\| ; y \in A\}, e(A, B):=\sup \{d(x, B) ; x \in A\}$ and $d_{H}(A, B)=$ $\max \{e(A, B), e(B, A)\}$. Let $K$ be a nonempty subset of $X$, for every $x^{*} \in X^{*}$, $\delta^{*}\left(x^{*}, K\right)=\sup \left\{\left\langle x^{*}, x\right\rangle: x \in K\right\}$ is called the support function of $K$.

Definition 1. ([1], Def. 2.2.1). A Banach space $X$ is said to be uniformly convex if for any $\varepsilon, 0<\varepsilon \leq 2$, the inequalities $\|x\| \leq 1$, $\|y\| \leq 1$ and $\|x-y\| \geq \varepsilon$ imply there exists $a \delta=\delta(\varepsilon)$ such that $\left\|\frac{1}{2}(x+y)\right\| \leq 1-\delta$.

Definition 2. A Banach space $X$ is said to be strictly convex if $x, y \in S_{X}$ with $x \neq y \Longrightarrow\|(1-\lambda) x+\lambda y\|<1$ for $0<\lambda<1$, where $S_{X}=\{z \in X:\|z\|=1\}$ is called the unit sphere on $X$.

Definition 3. ([1], Def. 2.4.1). Let $X^{*}$ be the topological dual of a Banach space $X$, then the set-valued map

$$
\begin{aligned}
J & : X \rightarrow 2^{X^{*}}, \\
J(x) & =\left\{y \in X^{*}:\langle x, y\rangle=\|x\|^{2}=\|y\|^{2}\right\}
\end{aligned}
$$

is said to be the normalized duality map (or duality map) in X. Moreover, the set-valued map

$$
\begin{aligned}
J^{*} & : X^{*} \rightarrow 2^{X}, \\
J^{*}(y) & =\left\{x \in X:\langle y, x\rangle=\|x\|^{2}=\|y\|^{2}\right\}
\end{aligned}
$$

is called the normalized duality map (or duality map) in $X^{*}$.

Many properties of the normalized duality map $J$ have been studied. For the details, one may consult [1, 3, 21]. We list in the following some properties of $J$ that we needed.

1. If $X$ is a Hilbert space, then $J(x)=\{x\}$ for all $x \in X$.

2. For each $x \in X, J(x)$ is nonempty closed convex and bounded subset of $X^{*}$.

3. $J(\lambda x)=\lambda J(x), \forall x \in X$ and $\forall \lambda \in \mathbb{R}$.

4. If $X^{*}$ is strictly convex, $J$ is single valued.

5. If $X$ is strictly convex, $J$ is one to one, i.e. $x \neq y \Rightarrow J(x) \cap J(y)=\phi$.

6. If $X^{*}$ is uniformly convex, then $J$ is uniformly continuous on each bounded set in $X$, i.e. $\forall \varepsilon>0$ and $\alpha>0$ there is a $\delta=\delta(\varepsilon, \alpha)>0$ such that

$$
\|x\| \leq \alpha,\|y\| \leq \alpha,\|x-y\|<\delta \Rightarrow\|J(x)-J(y)\|<\varepsilon .
$$

Note that if $X^{*}$ is uniformly convex, then it is strictly convex and hence $J$ is single-valued map. 
M.S. Abdo, A.G. Ibrahim, S.K. Panchal - Sweeping Process in Banach Spaces

7. If $X$ is reflexive, then $J$ is a map from $X$ onto $X^{*}$, that is

$$
\cup\{J(x): x \in X\}=X^{*} .
$$

8. If $X$ is reflexive strictly convex space with strictly convex conjugate space $X^{*}$, then $J$ and $J^{*}$ are one-to-one, onto and single-valued map and

$$
J^{-1}=J^{*}, J J^{*}=I_{X^{*}} \text { and } J^{*} J=I_{X},
$$

where $I_{X}$ is the identity map on $X$ and $I_{X^{*}}$ is the identity map on $X^{*}$.

Definition 4. ([1], Def. 2.8.1). The Banach space $X$ is said to be uniformly smooth if

$$
\rho_{X}^{\prime}(0)=\lim _{t \rightarrow 0} \frac{\rho_{X}(t)}{t}=0,
$$

where $\rho_{X}:[0, \infty[\longrightarrow[0, \infty[$ is called the modulus of smoothness of $X$, and defined by

$$
\begin{aligned}
\rho_{X}(t) & =\sup \left\{\frac{1}{2}(\|x+y\|+\|x-y\|)-1:\|x\|=1, \quad\|y\|=t\right\} \\
& =\sup \left\{\frac{1}{2}(\|x+t y\|+\|x-t y\|)-1:\|x\|=1, \quad\|y\|=1 ; t \geq 0\right\} .
\end{aligned}
$$

Lemma 1. ([1, 3, 21]). Let $X$ be a Banach space.

1. $X$ is uniformly smooth if and only if $X^{*}$ is uniformly convex.

2. $X$ is uniformly convex if and only if $X^{*}$ is uniformly smooth.

3. If $X$ is uniformly smooth, then $X$ is reflexive.

4. Every uniformly convex space is reflexive.

5. Every Hilbert space is uniformly convex.

6. Every uniformly convex space is strictly convex.

Now, let $X$ be a Banach space and $X^{*}$ be its topological dual. Let $V: X^{*} \times X \rightarrow$ $\mathbb{R}, V^{*}: X \times X^{*} \rightarrow \mathbb{R}$ be two functions defined by:

$$
\begin{aligned}
V(\varphi, x) & =\|\varphi\|^{2}-2\langle\varphi, x\rangle+\|x\|^{2}, \\
V^{*}(x, \varphi) & =V(\varphi, x) .
\end{aligned}
$$

Based on the functional $V$, the generalized projection of a point $\varphi$ in $X^{*}$ onto a nonempty subset $E$ of $X$ can be defined as: 
M.S. Abdo, A.G. Ibrahim, S.K. Panchal - Sweeping Process in Banach Spaces

Definition 5. ([3, 4]). Let $X$ be a Banach space, $E$ be a nonempty subset of $X$ and $\varphi \in X^{*}$. If there exists a point $z \in E$ satisfying

$$
V(\varphi, z)=d_{E}^{V}(\varphi)
$$

then $z$ is called a generalized projection of $\varphi$ onto $E$. Where $d_{E}^{V}(\varphi)=\inf _{x \in E} V(\varphi, x)$.

The set of all such points is denoted by $\pi_{E}(\varphi)$, i.e.

$$
\pi_{E}(\varphi)=\left\{z \in E: V(\varphi, z)=d_{E}^{V}(\varphi)\right\} \subseteq X .
$$

Now, we list in the following Lemma some properties of $V$ and $\pi_{E}(\varphi)$ (see [4]).

Lemma 2. Let $X$ be a Banach space and $X^{*}$ be its topological dual.

1. $V(J(x), x)=0$.

2. If $X$ is uniformly convex or uniformly smooth, then

$$
V(\varphi, x)=0 \Leftrightarrow \varphi=J(x), \forall x \in X \text { and } \varphi \in X^{*} .
$$

3. If $X$ is a Hilbert space, then

$$
V(\varphi, x)=\|\varphi-x\|^{2} .
$$

4. If $X$ is reflexive and $E$ is a nonempty closed and convex subset of $X$, then

(a) $\pi_{E}(\varphi) \neq \phi, \forall \varphi \in X^{*}$.

(b) $X$ is strictly convex if and only if $\pi_{E}(\varphi)$ is singleton for all $\varphi \in X^{*}$.

(c) If $X$ is also smooth, then for any given $\varphi \in X^{*}$,

$$
z \in \pi_{E}(\varphi) \Leftrightarrow\langle\varphi-J(z), x-z\rangle \leq 0, \forall x \in E .
$$

For more details about the convex normal cone (see $[12,13])$.

Lemma 3. ([13]). Let E be a nonempty, closed and convex subset of Banach space $X$ and $z \in E$. Then

1. $N_{E}(z) \cap \mathbb{B}^{*}=\partial d_{E}(z)$, where $\partial d_{E}(z)$ is the subdifferential of the function $z \rightarrow$ $d_{E}(z)$, and $d_{E}(z)$ is the distance from $z$ to $E$.

2. If $X$ is reflexive and smooth, then

$$
z \in \pi_{E}(\varphi) \Leftrightarrow \varphi-J(z) \in N_{E}(z), \forall \varphi \in X^{*} \text { and } \forall z \in X \text {. }
$$


M.S. Abdo, A.G. Ibrahim, S.K. Panchal - Sweeping Process in Banach Spaces

Lemma 4. ([8], Prop. 2.2). Let $\Omega$ be an open subset in a normal vector space $Z$, $X$ be a Banach space and $C$ be a Hausdorff continuous set-valued map defined on $\Omega$ and with nonempty compact convex values in $X$. Let $\left(\varphi_{n}\right)$ be a sequence of $X^{*}$ that converges weakly to $\varphi$ in $X^{*},\left(x_{n}\right)$ be a sequence in $X$ that converges strongly to $x$ in $X$ such that $\left(t_{n}\right)$ be a sequence in $\Omega$ that converges to $t$ in $\Omega$. If $\varphi_{n} \in \partial d_{C\left(t_{n}\right)}\left(x_{n}\right)$, then $\varphi \in \partial d_{C(t)}(x)$.

Definition 6. ([6], Def. 1, sec. 4, ch. 1). Let $X$ and $Y$ be two normed spaces. We say that a set-valued map $F: X \longrightarrow P(Y)-\{\phi\}$ is upper hemicontinuous at $x_{0} \in X$ if and only if for every $p \in Y^{*}$, the function $x \longrightarrow \delta^{*}(p, F(x))$ is upper semicontinuous at $x_{0} \in X$.

Obviously, if $F$ is upper semicontinuous at $x_{0}$, then it is upper hemicontinuous at $x_{0}$.

Theorem 5. ([6], Th. 1, sec. 4, ch. 1). (Convergence Theorem)

Let $F$ be a upper hemicontinuous map from a Hausdorff locally convex space $X$ to the closed convex subsets of a Banach space $Y$. Let I be an interval of $\mathbb{R}$ and $x_{k}($. and $y_{k}($.$) be measurable functions from I$ to $X$ and $Y$ respectively satisfying:

for almost all $t \in I$, for every neighborhood $\mathcal{N}$ of 0 in $X \times Y$ there exists $k_{0}=k_{0}(t, \mathcal{N})$ such that $\forall k \geq k_{0},\left(x_{k}(t), y_{k}(t)\right) \in \operatorname{graph}(F)+\mathcal{N}$. If

(i) $x_{k}($.$) converges almost everywhere to a function x($.$) from I$ to $X$,

(ii) $y_{k}(.) \in L^{1}(I, Y)$ and converges weakly to $y($.$) in L^{1}(I, Y)$,

then for almost all $t \in I, \quad(x(t), y(t)) \in \operatorname{graph}(F)$, i.e. $y(t) \in F(x(t))$.

Lemma 6. ([24], Th. 2.2). Let $\Omega$ be a subset of a Banach space $X$. Assume that $G:[a, b] \times \Omega \rightarrow 2^{X}$ is a set-valued map such that

1. for every $x \in \Omega$, the set-valued map $G(., x)$ is measurable on $[a, b]$.

2. for every $t \in[a, b]$, the set-valued map $G(t,$.$) is continuous on \Omega$.

Then for any measurable function $x():.[a, b] \rightarrow \Omega$, the set-valued map $G(., x()$. is measurable on $[a, b]$.

Lemma 7. ([24], Lem. 2.3). Let $X$ be a separable Banach space, $F:[a, b] \rightarrow 2^{X} a$ measurable set-valued map and $z:[a, b] \rightarrow X$ a measurable function. Then for any positive measurable function $r:[a, b] \rightarrow \mathbb{R}^{+}$, there exists a measurable selection $f$ of $F$ such that for almost $t \in[a, b]$

$$
\|f(t)-z(t)\| \leq d(z(t), F(t))+r(t) .
$$


M.S. Abdo, A.G. Ibrahim, S.K. Panchal - Sweeping Process in Banach Spaces

\section{MAIN RESULT}

In this section, we prove the existence result for the probelm 2 .

Theorem 8. Let $X$ be a separable uniformly convex and uniformly smooth Banach space, $I=[0, T](T>0), C$ be a set-valued map defined from $I \times X$ to the family of nonempty compact convex subsets of $X$ and $G$ be a set-valued map defined from $I \times \mathcal{C}_{r} \times \mathcal{C}_{r}$ with nonempty closed values in $X^{*}$. Assume that the following hypotheses hold:

$\left(C_{1}\right) C$ is Hausdorff continuous;

$\left(C_{2}\right)$ there is a compact convex subset $K$ of $X$ such that

$$
C(t, x) \subset K, \forall(t, x) \in I \times X
$$

$\left(G_{1}\right)$ for each $f, g \in \mathcal{C}_{r}, t \rightarrow G(t, f, g)$ is a measurable;

$\left(G_{2}\right)$ there is a nondecreasing continuous function $\Lambda: I \rightarrow \mathbb{R}^{+}$such that for all $t, s \in I$, and for all $\psi_{1}, \varphi_{1}, \psi_{2}, \varphi_{2} \in \mathcal{C}_{r}$

$$
d_{H}\left(G\left(t, \psi_{1}, \varphi_{1}\right), G\left(s, \psi_{2}, \varphi_{2}\right)\right) \leq|\Lambda(s)-\Lambda(t)|\left(\left\|\psi_{1}-\psi_{2}||+\right\| \varphi_{1}-\varphi_{2} \|\right) ;
$$

$\left(G_{3}\right)$ there is a continuous function $p: I \rightarrow \mathbb{R}^{+}$, such that for all $f, g \in \mathcal{C}_{r}$,

$$
\|G(t, f, g)\| \leq p(t)(1+\|f(0)\|+\|g(0)\|) \text {, a.e. } t \in I \text {. }
$$

Then for any $\varphi, \psi \in \mathcal{C}_{r}$ with $\varphi(0) \in C(0, \psi(0))$, there exist two continuous functions $u:[-r, T] \rightarrow X, w:[-r, T] \rightarrow X$ such that $u$ and $w$ are absolutely continuous functions on $I$ and that (2) is satisfied.

Proof. At first, we note that, since the Banach space $X$ is uniformly convex, then by Lemma 1 , it is reflexive and strictly convex. Moreover, since $X$ is uniformly smooth then, again by Lemma $1, X^{*}$ is uniformly convex, and hence $X^{*}$ is strictly convex. Then, by properties (6) and (8) which follow Definition $3, J$ and $J^{*}$ are one to one, onto, single-valued maps and $J J^{*}=I_{X^{*}}$ and $J^{*} J=I_{X}$. Furthermore, $J$ is uniformly continuous on bounded sets. Let $k>0$ be such that $K \subset k \mathbb{B}$, and $\mu=\beta(1+\|\psi(0)\|+4 k)$ where $\beta=\max _{t \in I}|p(t)|$.

For notational convenience, we take $T=1$. In order to make it easier for the reader we will divide the proof into the following steps:

Step 1. In this step we prove that for each natural number $m$ and for each $z \in$ $L^{1}\left(I, X^{*}\right)$, there exist two continuous functions $u_{m}, w_{m}:[-r, 1] \rightarrow X, g_{m} \in L^{1}\left(I, X^{*}\right)$ 
M.S. Abdo, A.G. Ibrahim, S.K. Panchal - Sweeping Process in Banach Spaces

and two step functions $\theta_{m}, \delta_{m}: I \rightarrow I$ such that $u_{m}$ and $w_{m}$ are absolutely continuous functions on $I, \lim _{m \rightarrow \infty} \theta_{m}(t)=\lim _{m \rightarrow \infty} \delta_{m}(t)=t$ and that

(i) $u_{m}(t)=\varphi(t), w_{m}(t)=\psi(t)$, for $t \in[-r, 0]$;

(ii) $g_{m}(t) \in G\left(t, \tau\left(\delta_{m}(t)\right) w_{m}, \tau\left(\delta_{m}(t)\right) u_{m}\right)$, for $t \in I$;

$($ iii $)\left\|g_{m}(t)-z(t)\right\| \leq d\left(z(t), G\left(t, \tau\left(\delta_{m}(t)\right) w_{m}, \tau\left(\delta_{m}(t)\right) u_{m}\right)\right)$

$+\frac{1}{m^{2}}$, for $t \in I$

(iv) $w_{m}(t)=w_{m}\left(\delta_{m}(t)\right)+\left(t-\delta_{m}(t)\right) u_{m}\left(\delta_{m}(t)\right)$, for $t \in I$;

(v) $u_{m}(t)=\varphi(0)+\int_{0}^{t} u_{m}^{\prime}(s) d s$, for all $t \in I$

(vi) $u_{m}^{\prime}(t) \in C\left(\theta_{m}(t), w_{m}\left(\theta_{m}(t)\right)\right) \cap \mathbb{B}(0, k)$, for a.e. $t \in I$;

(vii) $\left\|u_{m}\left(\delta_{m}(t)\right)\right\| \leq 2 k$, for $t \in I$

(viii) $\left\|J\left(u_{m}^{\prime}(t)\right)-g_{m}\left(\delta_{m}(t)\right)\right\| \leq k+\mu$, a.e. for $t \in I$;

(ix) $J\left(u_{m}^{\prime}(t)\right)-g_{m}\left(\delta_{m}(t)\right) \in-N_{C\left(\theta_{m}(t), w_{m}\left(\theta_{m}(t)\right)\right)}\left(u_{m}^{\prime}(t)\right)$, a.e. for $t \in I$.

Consider a partition of $I=[0,1]$ by the points $t_{i}^{m}=i e_{m}, e_{m}=\frac{1}{m}, 0 \leq i \leq m$. Let $\left.\left.I_{0}^{m}=\left\{t_{0}^{m}\right\}=\{0\}, I_{i+1}^{m}=\right] t_{i}^{m}, t_{i+1}^{m}\right], 0 \leq i \leq m-1$. For $t \in[-r, 0]$, we put $u_{m}(t):=\varphi(t)$ and $w_{m}(t):=\psi(t)$. Note that, in view of Lemma 6 and Lemma 7 , there exists a function $g_{0}^{m} \in L^{1}\left(\left[t_{0}^{m}, t_{1}^{m}\right], X^{*}\right)$ such that $g_{0}^{m}(t) \in G\left(t, \tau\left(t_{0}^{m}\right) \psi, \tau\left(t_{0}^{m}\right) \varphi\right)$ and

$$
\left\|g_{0}^{m}(t)-z(t)\right\| \leq d(z(t), G(t, \tau(0) \psi, \tau(0) \varphi))+\frac{1}{m^{2}}, \text { for all } t \in\left[t_{0}^{m}, t_{1}^{m}\right] .
$$

Set $x_{0}^{m}=\varphi(0) \in C(0, \psi(0))$ and for $t \in\left[t_{0}^{m}, t_{1}^{m}\right]$ we define

$$
w_{m}(t):=\psi\left(t_{0}^{m}\right)+\left(t-t_{0}^{m}\right) x_{0}^{m},
$$

and

$$
u_{m}(t):=\frac{t_{1}^{m}-t}{e_{m}} x_{0}^{m}+\frac{t-t_{0}^{m}}{e_{m}} x_{1}^{m}
$$

where

$$
x_{1}^{m}:=x_{0}^{m}+e_{m} \pi_{C\left(t_{1}^{m}, w_{m}\left(t_{1}^{m}\right)\right)}\left(g_{0}^{m}\left(t_{0}^{m}\right)\right) .
$$

Note that $x_{1}^{m}$ is well defined since the generalized projection $\pi$ exists by Lemma 2. From the definition of $u_{m}$, the choice $x_{1}^{m}$ and $\left(C_{2}\right)$ we get, by Lemma 3 , for $t \in] t_{0}^{m}, t_{1}^{m}[$

$$
u_{m}^{\prime}(t)=\frac{x_{1}^{m}-x_{0}^{m}}{e_{m}} \in C\left(t_{1}^{m}, w_{m}\left(t_{1}^{m}\right)\right) \cap \mathbb{B}(0, k),
$$

and

$$
J\left(u_{m}^{\prime}(t)\right)-g_{0}^{m}\left(t_{0}^{m}\right) \in-N_{C\left(t_{1}^{m}, w_{m}\left(t_{1}^{m}\right)\right)}\left(u_{m}^{\prime}(t)\right) .
$$

Now by Lemma 6 , Lemma 7 and by induction we can define

$$
w_{m}(t):=w_{m}\left(t_{i}^{m}\right)+\left(t-t_{i}^{m}\right) u_{m}\left(t_{i}^{m}\right), t \in I_{i+1}^{m},
$$


and

$$
u_{m}(t):=\frac{t_{i+1}^{m}-t}{e_{m}} x_{i}^{m}+\frac{t-t_{i}^{m}}{e_{m}} x_{i+1}^{m}, t \in I_{i+1}^{m},
$$

where

$$
x_{i+1}^{m}:=x_{i}^{m}+e_{m} \pi_{C\left(t_{i+1}^{m}, w_{m}\left(t_{i+1}^{m}\right)\right)}\left(g_{i}^{m}\left(t_{i}^{m}\right)\right),
$$

and for any $\left.\left.1 \leq i \leq m-1, g_{i}^{m} \in L^{1}(] t_{i}^{m}, t_{i+1}^{m}\right], X^{*}\right)$ such that

$$
g_{i}^{m}(t) \in G\left(t, \tau\left(t_{i}^{m}\right) w_{m}, \tau\left(t_{i}^{m}\right) u_{m}\right),
$$

and

$$
\left.\left.\left\|g_{i}^{m}(t)-z(t)\right\| \leq d\left(z(t), G\left(t, \tau\left(t_{i}^{m}\right) w_{m}, \tau\left(t_{i}^{m}\right) u_{m}\right)\right)+\frac{1}{m^{2}} \text {, for all } t \in\right] t_{i}^{m}, t_{i+1}^{m}\right] .
$$

The existence of such $g_{i}^{m}$ is ensured by Lemma 6. Moreover, let $\theta_{m}(0)=0$, $\theta_{m}(t)=t_{i+1}^{m}$, for all $\left.\left.t \in\right] t_{i}^{m}, t_{i+1}^{m}\right](0 \leq i \leq m-1), \delta_{m}(t)=t_{i}^{m}$ for all $t \in\left[t_{i}^{m}, t_{i+1}^{m}[(0 \leq\right.$ $i \leq m-1)$ and $\delta_{m}(1)=t_{m}^{m}$. Clearly $w_{m}$ and $u_{m}$ are absolutely continuous on $I$ and differentiable on $I \backslash\left\{t_{i}^{m} ; i=0,1,2, \ldots, m\right\}$ and for all $\left.t \in\right] t_{i}^{m}, t_{i+1}^{m}[$

$$
u_{m}^{\prime}(t)=\frac{x_{i+1}^{m}-x_{i}^{m}}{e_{m}} \in C\left(t_{i+1}^{m}, w_{m}\left(t_{i+1}^{m}\right)\right) \cap \mathbb{B}(0, k) .
$$

On the other hand, by recalling the definition of the generalized projection we have

$$
J\left(u_{m}^{\prime}(t)\right)-g_{i}^{m}\left(t_{i}^{m}\right) \in-N_{C\left(t_{i+1}^{m}, w_{m}\left(t_{i+1}^{m}\right)\right)}\left(u_{m}^{\prime}(t)\right) \text {, for a.e. } t \in I_{i+1}^{m} .
$$

Next, let us define a function $g_{m}: I \rightarrow X^{*}$ as $g_{m}(t)=g_{0}^{m}(t)$ for $t \in\left[t_{0}^{m}, t_{1}^{m}\right]$ and $g_{m}(t)=g_{i}^{m}(t)$ for $\left.\left.t \in\right] t_{i}^{m}, t_{i+1}^{m}\right], i=1,2, \ldots, m-1$.

Then, from (4), (7), (8), (9) and definitions of $\theta_{m}, \delta_{m}$ and $g_{m}$, one obtains

$$
\begin{gathered}
w_{m}(t)=w_{m}\left(\delta_{m}(t)\right)+\left(t-\delta_{m}(t)\right) u_{m}\left(\delta_{m}(t)\right), \text { for } t \in I, \\
u_{m}^{\prime}(t) \in C\left(\theta_{m}(t), w_{m}\left(\theta_{m}(t)\right)\right) \cap \mathbb{B}(0, k), \text { for a.e. } t \in I, \\
J\left(u_{m}^{\prime}(t)\right)-g_{m}\left(\delta_{m}(t)\right) \in-N_{C\left(\theta_{m}(t), w_{m}\left(\theta_{m}(t)\right)\right)}\left(u_{m}^{\prime}(t)\right), \text { for a.e. } t \in I, \\
g_{m}(t) \in G\left(t, \tau\left(\delta_{m}(t)\right) w_{m}, \tau\left(\delta_{m}(t)\right) u_{m}\right), \text { for } t \in I,
\end{gathered}
$$

and

$$
\left\|g_{m}(t)-z(t)\right\| \leq d\left(z(t), G\left(t, \tau\left(\delta_{m}(t)\right) w_{m}, \tau\left(\delta_{m}(t)\right) u_{m}\right)\right)+\frac{1}{m^{2}}, t \in I .
$$

In order to prove the properties (vii) and (viii) of (3) we observe that, from (11) for any $i=0,1,2, \ldots, m-1$ and for any $t \in] t_{i}^{m}, t_{i+1}^{m}[$

$$
\left\|\frac{x_{i+1}^{m}-x_{i}^{m}}{e_{m}}\right\|=\left\|u_{m}^{\prime}(t)\right\| \leq k .
$$


M.S. Abdo, A.G. Ibrahim, S.K. Panchal - Sweeping Process in Banach Spaces

So,

$$
\left\|x_{i+1}^{m}\right\| \leq\left\|x_{i}^{m}\right\|+e_{m} k, \forall i=0,1,2, \ldots, m-1 .
$$

Then

$$
\begin{aligned}
\left\|x_{i+1}^{m}\right\| & \leq\left\|x_{i}^{m}\right\|+e_{m} k \\
& \leq\left\|x_{i-1}^{m}\right\|+2 e_{m} k .
\end{aligned}
$$

We reiterate this process to get

$$
\begin{aligned}
\left\|x_{i+1}^{m}\right\| & \leq\left\|x_{0}^{m}\right\|+(i+1) e_{m} k \\
& \leq 2 k .
\end{aligned}
$$

So, (vii) is true. Also, from (4) and (5) for $i=0,1,2, \ldots, m-1$ we have

$$
\begin{aligned}
\left\|w_{m}\left(t_{i+1}^{m}\right)\right\| & \leq\left\|w_{m}\left(t_{i}^{m}\right)\right\|+\left|t_{i+1}^{m}-t_{i}^{m}\right|\left\|u_{m}\left(t_{i}^{m}\right)\right\| \\
& =\left\|w_{m}\left(t_{i}^{m}\right)\right\|+\left|t_{i+1}^{m}-t_{i}^{m}\right|\left\|x_{i}^{m}\right\| \\
& \leq\left\|w_{m}\left(t_{i}^{m}\right)\right\|+2 e_{m} k \\
& \leq\left\|w_{m}\left(t_{i-1}^{m}\right)\right\|+4 e_{m} k .
\end{aligned}
$$

We reiterate this process to get

$$
\begin{aligned}
\left\|w_{m}\left(t_{i+1}^{m}\right)\right\| & \leq\|\psi(0)\|+2 m e_{m} k \\
& =\|\psi(0)\|+2 k .
\end{aligned}
$$

This inquality with the condition $\left(G_{3}\right)$, the property $(v i i)$ of $(3)$ and (13) for $i=0,1,2, \ldots, m-1$, we have

$$
\begin{aligned}
\left\|g_{m}(t)\right\| & \leq p(t)\left(1+\left\|\left(\tau\left(\delta_{m}(t)\right) w_{m}\right)(0)\right\|+\left\|\left(\tau\left(\delta_{m}(t)\right) u_{m}\right)(0)\right\|\right) \\
& =p(t)\left(1+\left\|w_{m}\left(\delta_{m}(t)\right)\right\|+\left\|u_{m}\left(\delta_{m}(t)\right)\right\|\right) \\
& \leq p(t)(1+\|\psi(0)\|+2 k+2 k) \\
& =\beta(1+\|\psi(0)\|+4 k) \\
& =\mu
\end{aligned}
$$

Therefore, for $t \in I \backslash\left\{t_{i}^{m} ; i=0,1,2, \ldots, m\right\}$

$$
\begin{aligned}
\left\|J\left(u_{m}^{\prime}(t)\right)-g_{m}\left(\delta_{m}(t)\right)\right\| & \leq\left\|J\left(u_{m}^{\prime}(t)\right)\right\|+\left\|g_{m}\left(\delta_{m}(t)\right)\right\| \\
& \leq\left\|u_{m}^{\prime}(t)\right\|+\mu \\
& \leq k+\mu .
\end{aligned}
$$


M.S. Abdo, A.G. Ibrahim, S.K. Panchal - Sweeping Process in Banach Spaces

So, (viii) is satisfied and our goal in this step has been achieved.

Step 2. In this step we prove that for each natural number $n$ there are two continuous functions $u_{n}, w_{n}:[-r, 1] \rightarrow X, g_{n} \in L^{1}\left(I, X^{*}\right)$ and $\theta_{n}, \delta_{n} \in S(I, I)$, where $S(I, I)$ denotes the space of step functions from $I$ to $I$, such that $u_{n}$ and $w_{n}$ are absolutely continuous functions on $I, \lim _{n \rightarrow \infty} \theta_{n}(t)=\lim _{n \rightarrow \infty} \delta_{n}(t)=t$ and that

(i) $u_{n}(t)=\varphi(t), w_{n}(t)=\psi(t)$, for $t \in[-r, 0]$;

(ii) $g_{n}(t) \in G\left(t, \tau\left(\delta_{n}(t)\right) w_{n}, \tau\left(\delta_{n}(t)\right) u_{n}\right)$, for $t \in I$;

$($ iii $)\left\|g_{n+1}(t)-g_{n}(t)\right\| \leq d\left(g_{n}(t), G\left(t, \tau\left(\delta_{n+1}(t)\right) w_{n+1}, \tau\left(\delta_{n+1}(t)\right) u_{n+1}\right)\right)+$ $\frac{1}{(n+1)^{2}}, \forall n \geq 1$, for $t \in I$;

(iv) $w_{n}(t)=w_{n}\left(\delta_{n}(t)\right)+\left(t-\delta_{n}(t)\right) u_{n}\left(\delta_{n}(t)\right)$, for $t \in I$;

(v) $u_{n}(t)=\varphi(0)+\int_{0}^{t} u_{n}^{\prime}(s) d s$, for all $t \in I$

(vi) $u_{n}^{\prime}(t) \in C\left(\theta_{n}(t), w_{n}\left(\theta_{n}(t)\right)\right) \cap \mathbb{B}(0, k)$, for a.e. $t \in I$;

(vii) $\left\|u_{n}\left(\delta_{n}(t)\right)\right\| \leq 2 k$, for $t \in I$;

(viii) $\left\|J\left(u_{n}^{\prime}(t)\right)-g_{n}\left(\delta_{n}(t)\right)\right\| \leq k+\mu$, a.e. for $t \in I$;

$(i x) J\left(u_{n}^{\prime}(t)\right)-g_{n}\left(\delta_{n}(t)\right) \in-N_{C\left(\theta_{n}(t), w_{n}\left(\theta_{n}(t)\right)\right.}\left(u_{n}^{\prime}(t)\right)$, a.e. for $t \in I$.

We use the induction to prove our goal in this step. From Step 1 for $m=1$ there are two continuous functions $u_{1}, w_{1}:[-r, 1] \rightarrow X, g_{1} \in L^{1}\left(I, X^{*}\right)$ and $\theta_{1}$, $\delta_{1} \in S(I, I)$ such that $u_{1}$ and $w_{1}$ are absolutely continuous functions on $I$ and all the properties $(i),(i i),(i v) \rightarrow(i x)$ in the relation (16) are satisfied.

Next, assume that for each $m \leq n$ there are two continuous functions $u_{m}, w_{m}$ : $[-r, 1] \rightarrow X, g_{m} \in L^{1}\left(I, X^{*}\right)$ and $\theta_{m}, \delta_{m} \in S(I, I)$ such that $u_{m}$ and $w_{m}$ are absolutely continuous functions on $I$ and all the properties $(i),(i i),(i v) \rightarrow(i x)$ in the relation (16) are satisfied for each $m \leq n$ and

$$
\begin{aligned}
& \left\|g_{m}(t)-g_{m-1}(t)\right\| \\
\leq & d\left(g_{m-1}(t), G\left(t, \tau\left(\delta_{m}(t)\right) w_{m}, \tau\left(\delta_{m}(t)\right) u_{m}\right)\right)+\frac{1}{m^{2}}, \forall m=2, \ldots, n .
\end{aligned}
$$

In order to define $u_{n+1}, w_{n+1}, g_{n+1}, \theta_{n+1}$ and $\delta_{n+1}$ we consider a partition for $I$ by the points $t_{i}^{n+1}=i e_{n+1}, e_{n+1}=\frac{1}{n+1}, 0 \leq i \leq n+1$. Let $I_{0}^{n+1}=\left\{t_{0}^{n+1}\right\}=\{0\}$, $\left.\left.I_{i+1}^{n+1}=\right] t_{i}^{n+1}, t_{i+1}^{n+1}\right], 0 \leq i \leq n$. For $t \in[-r, 0]$, we put $u_{n+1}(t):=\varphi(t)$ and $w_{n+1}(t):=\psi(t)$. Note that, in view of Lemma 6 and Lemma 7 , there exists a function $g_{0}^{n+1} \in L^{1}\left(\left[t_{0}^{n+1}, t_{1}^{n+1}\right], X^{*}\right)$ such that $g_{0}^{n+1}(t) \in G(t, \tau(t(0)) \psi, \tau(t(0)) \varphi)$ and for all $t \in\left[t_{0}^{n+1}, t_{1}^{n+1}\right]$

$$
\left\|g_{0}^{n+1}(t)-g_{n}(t)\right\| \leq d\left(g_{n}(t), G(t, \tau(0) \psi, \tau(0) \varphi)\right)+\frac{1}{(n+1)^{2}} .
$$

Set $x_{0}^{n+1}=\varphi(0) \in C(0, \psi(0))$ and for $t \in\left[t_{0}^{n+1}, t_{1}^{n+1}\right]$ we define

$$
w_{n+1}(t):=\psi\left(t_{0}^{n+1}\right)+\left(t-t_{0}^{n+1}\right) x_{0}^{n+1},
$$


and

$$
u_{n+1}(t):=\frac{t_{1}^{n+1}-t}{e_{n+1}} x_{0}^{n+1}+\frac{t-t_{0}^{n+1}}{e_{n+1}} x_{1}^{n+1},
$$

where

$$
x_{1}^{n+1}:=x_{0}^{n+1}+e_{n+1} \pi_{C\left(t_{1}^{n+1}, w_{n+1}\left(t_{1}^{n+1}\right)\right)}\left(g_{0}^{n+1}\left(t_{0}^{n+1}\right)\right) .
$$

Next, as in Step 1, for $t \in] t_{0}^{n+1}, t_{1}^{n+1}[$

$$
u_{n+1}^{\prime}(t)=\frac{x_{1}^{n+1}-x_{0}^{n+1}}{e_{n+1}} \in C\left(t_{1}^{n+1}, w_{n+1}\left(t_{1}^{n+1}\right)\right) \cap \mathbb{B}(0, k),
$$

and

$$
J\left(u_{n+1}^{\prime}(t)\right)-g_{0}^{n+1}\left(t_{0}^{n+1}\right) \in-N_{C\left(t_{1}^{n+1}, w_{n+1}\left(t_{1}^{m}\right)\right)}\left(u_{n+1}^{\prime}(t)\right) .
$$

Also, by induction we can define

$$
w_{n+1}(t):=w_{n+1}\left(t_{i}^{n+1}\right)+\left(t-t_{i}^{n+1}\right) u_{n+1}\left(t_{i}^{n+1}\right), t \in I_{i+1}^{n+1},
$$

and

$$
u_{n+1}(t):=\frac{t_{i+1}^{n+1}-t}{e_{n+1}} x_{i}^{n+1}+\frac{t-t_{i}^{n+1}}{e_{n+1}} x_{i+1}^{n+1}, t \in I_{i+1}^{n+1},
$$

where

$$
x_{i+1}^{n+1}:=x_{i}^{n+1}+e_{n+1} \pi_{C\left(t_{i+1}^{n+1}, w_{n+1}\left(t_{i+1}^{n+1}\right)\right)}\left(g_{i}^{n+1}\left(t_{i}^{n+1}\right)\right),
$$

and for any $\left.\left.1 \leq i \leq n, g_{i}^{n} \in L^{1}(] t_{i}^{n+1}, t_{i+1}^{n+1}\right], X^{*}\right)$ such that

$$
g_{i}^{n+1}(t) \in G\left(t, \tau\left(t_{i}^{n+1}\right) w_{n+1}, \tau\left(t_{i}^{n+1}\right) u_{n+1}\right),
$$

and

$$
\left\|g_{i}^{n+1}(t)-g_{n}(t)\right\| \leq d\left(g_{n}(t), G\left(t, \tau\left(t_{i}^{n+1}\right) w_{n+1}, \tau\left(t_{i}^{n+1}\right) u_{n+1}\right)\right)+\frac{1}{(n+1)^{2}},
$$

for all $\left.t \in] t_{i}^{n+1}, t_{i+1}^{n+1}\right]$.

Now we define $g_{n+1}: I \rightarrow X^{*}$ as $g_{n+1}(t)=g_{0}^{n+1}(t)$ for $t \in\left[t_{0}^{n+1}, t_{1}^{n+1}\right]$ and $g_{n+1}(t)=g_{i}^{n+1}(t)$ for $\left.\left.t \in\right] t_{i}^{n+1}, t_{i+1}^{n+1}\right], \quad 1 \leq i \leq n$. Moreover, $\theta_{n+1}$ and $\delta_{n+1}$ are defined as follows: $\theta_{n+1}(0)=0, \theta_{n+1}(t)=t_{i+1}^{n+1}$ for all $\left.] t_{i}^{n+1}, t_{i+1}^{n+1}\right](0 \leq i \leq n)$ $\delta_{n+1}(t)=t_{i}^{n+1}$ for all $\left[t_{i}^{n+1}, t_{i+1}^{n+1}\left[(0 \leq i \leq n)\right.\right.$ and $\delta_{n+1}(1)=t_{n+1}^{n+1}$.

By arguing as in Step 1 we can show that the functions $u_{n+1}, w_{n+1}, g_{n+1}, \theta_{n+1}$ and $\delta_{n+1}$ satisfy all the properties $(i) \rightarrow(i x)$ in (16).

Step 3. In this step we show that the sequences $\left(u_{n}\right)$ and $\left(w_{n}\right)$ have subsequences (still denoted by $\left(u_{n}\right)$ and $\left(w_{n}\right)$ ) converging uniformly to continuous functions $u$ : 
$[-r, 1] \rightarrow X, w:[-r, 1] \rightarrow X$, respectively, such that $u(t)=\varphi(t), w(t)=\psi(t)$ for $t \in[-r, 0] u$ and $w$ are absolutely continuous on $[0,1]$ and $u^{\prime}(t) \in C(t, w(t))$ for a.e. $t \in I$.

Indeed, by the property (vi) of (16) for $t, s \in I(t<s)$ and for any $n \geq 1$, we get

$$
\left\|u_{n}(s)-u_{n}(t)\right\| \leq \int_{t}^{s}\left\|u_{n}^{\prime}(\eta)\right\| d \eta \leq k|s-t|,
$$

which means that the set $\left\{u_{n}: n \geq 1\right\}$ is equicontinuous on $I$.

Moreover, by the property (vi) of (16) for any $n \geq 1$ and almost for $t \in I$, we have

$$
u_{n}^{\prime}(t) \in C\left(\theta_{n}(t), w_{n}\left(\theta_{n}(t)\right)\right)
$$

which implies

$$
u_{n}^{\prime}(t) \in K, \text { a.e. } t \in I \text {. }
$$

Hence, for all $t \in I$ $u_{n}(t)=\varphi(0)+\int_{0}^{t} u_{n}^{\prime}(s) d s \in \varphi(0)+I \times K=L$ which is compact.

Then, for any $t \in I$, the set $\left\{u_{n}(t): n \geq 1\right\}$ is relatively compact subset in $X$. Thus, by Arzela-Ascoli's Theorem, we can select a subsequence of $u_{n}$, again denoted by $u_{n}$, which converges uniformly to a continuous function $u$ on $I$.

Note that, because $\left(u_{n}^{\prime}\right)$ is uniformly bounded, we can extract a subsequence (still denoted by $\left.\left(u_{n}^{\prime}\right)\right)$, converging $\sigma\left(L^{\infty}(I, X), L^{1}\left(I, X^{*}\right)\right)$ to a function $z \in L^{\infty}(I, X)$. This means that for any $h \in L^{1}\left(I, X^{*}\right)$ we have $\lim _{n \rightarrow \infty}\left\langle h, u_{n}^{\prime}\right\rangle=\langle h, z\rangle$.

Since $L^{\infty}\left(I, X^{*}\right) \subseteq L^{1}\left(I, X^{*}\right)$, then $\lim _{n \rightarrow \infty}\left\langle h, u_{n}^{\prime}\right\rangle=\langle h, z\rangle, \forall h \in L^{\infty}\left(I, X^{*}\right)$. That is $u_{n}^{\prime} \rightarrow z$ in $\sigma\left(L^{1}(I, X), L^{\infty}\left(I, X^{*}\right)\right)$.

Now, for any $t \in I$ and any $v \in X^{*}$, consider the function $h=v \chi_{[0, t]}$. Then

$$
\begin{aligned}
\lim _{n \rightarrow \infty} v\left(\int_{0}^{t} u_{n}^{\prime}(s) d s\right) & =\lim _{n \rightarrow \infty} \int_{0}^{t} v\left(u_{n}^{\prime}(s)\right) d s \\
& =\lim _{n \rightarrow \infty}\left\langle h, u_{n}^{\prime}\right\rangle \\
& =\langle h, z\rangle \\
& =v\left(\int_{0}^{t} z(s) d s\right) .
\end{aligned}
$$

This means that for any $t \in I$ the sequence $\int_{0}^{t} u_{n}^{\prime}(s) d s$ converges weakly to $\int_{0}^{t} z(s) d s$ in $X$, and hence for all $t \in I$

$$
\lim _{n \rightarrow \infty} u_{n}(t)=\varphi(0)+\int_{0}^{t} u_{n}^{\prime}(s) d s
$$


weakly in $X$. But $\lim _{n \rightarrow \infty} u_{n}(t)=u(t)$, for all $t \in I$. Then

$$
u(t)=\varphi(0)+\int_{0}^{t} z(s) d s, \forall t \in I .
$$

This equality implies $u^{\prime}(t)=z(t)$, a.e. Therefore, $u_{n}^{\prime}$ converges to $u^{\prime}$ in $\sigma\left(L^{1}(I, X), L^{\infty}\left(I, X^{*}\right)\right)$ and $\mathrm{u}$ is absolutely continuous function. That is

$$
u(t)=\varphi(0)+\int_{0}^{t} u^{\prime}(s) d s, \forall t \in I .
$$

Now let $t \in I$ be any fixed point. Then there is a natural number $n$ such that $t \in I_{i+1}^{n}$ for some $i \in\{0,1,2, \ldots, n-1\}$. According to the definition of $w_{n}$ one obtains

$$
\begin{aligned}
w_{n}(t) & =w_{n}\left(t_{i}^{n}\right)+\left(t-t_{i}^{n}\right) u_{n}\left(t_{i}^{n}\right) \\
& =w_{n}\left(t_{i}^{n}\right)+\int_{t_{i}^{n}}^{t} u_{n}\left(\delta_{n}(s)\right) d s \\
& =w_{n}\left(t_{i-1}^{n}\right)+\left(t_{i}^{n}-t_{i-1}^{n}\right) u_{n}\left(t_{i-1}^{n}\right)+\int_{t_{i}^{n}}^{t} u_{n}\left(\delta_{n}(s)\right) d s \\
& =w_{n}\left(t_{i-1}^{n}\right)+\int_{t_{i-1}^{n}}^{t_{i}^{n}} u_{n}\left(\delta_{n}(s)\right) d s+\int_{t_{i}^{n}}^{t} u_{n}\left(\delta_{n}(s)\right) d s .
\end{aligned}
$$

We reiterate this process to get

$$
w_{n}(t)=\psi(0)+\int_{0}^{t} u_{n}\left(\delta_{n}(s)\right) d s .
$$

Next, let $w: I \rightarrow X$ be such that $w(t)=\psi(0)+\int_{0}^{t} u(s) d s$. For any $t \in I$, we have

$$
\begin{aligned}
\left\|w_{n}(t)-w(t)\right\| & \leq \int_{0}^{t}\left\|u_{n}\left(\delta_{n}(s)\right)-u(s)\right\| d s \\
& \leq \int_{0}^{t}\left\|u_{n}\left(\delta_{n}(s)\right)-u_{n}(s)\right\| d s+\int_{0}^{t}\left\|u_{n}(s)-u(s)\right\| d s \\
& \leq \sup _{s \in I}\left\|u_{n}\left(\delta_{n}(s)\right)-u_{n}(s)\right\|+\sup _{s \in I}\left\|u_{n}(s)-u(s)\right\| \\
& \leq k \sup _{s \in I}\left|\delta_{n}(s)-s\right|+\left\|u_{n}-u\right\|_{\infty} \\
& \leq \frac{k}{n}+\left\|u_{n}-u\right\|_{\infty}
\end{aligned}
$$

This shows that $\left(w_{n}\right)$ converges uniformly to $w$. 
Now, from (17) and Theorem 5 we infer that $u^{\prime}(t) \in C(t, w(t))$ for a.e. $t \in I$. Step 4. Our aim in this step is to show that for any $t \in I$, the sequence $\tau\left(\delta_{n}(t) u_{n}\right.$ converges to $\tau(t) u$ and the sequence $\tau\left(\delta_{n}(t) w_{n}\right.$ converges to $\tau(t) w$ in $\mathcal{C}_{r}$.

Let $t \in I$, we have

$$
\begin{aligned}
& \left\|\tau\left(\delta_{n}(t)\right) u_{n}-\tau(t) u\right\| \\
& \leq\left\|\tau\left(\delta_{n}(t)\right) u_{n}-\tau(t) u_{n}\right\|+\left\|\tau(t) u_{n}-\tau(t) u\right\| \\
& \leq \sup _{-r \leq s \leq 0}\left\|u_{n}\left(\delta_{n}(t)+s\right)-u_{n}(t+s)\right\|+\left\|\tau(t) u_{n}-\tau(t) u\right\| \\
& \leq \quad \sup \quad\left\|u_{n}\left(s_{1}\right)-u_{n}\left(s_{2}\right)\right\|+\left\|\tau(t) u_{n}-\tau(t) u\right\| \\
& -r \leq s_{1} \leq s_{2} \leq 1 \\
& \left|s_{1}-s_{2}\right| \leq \frac{1}{n} \\
& \leq \quad \sup \quad\left\|u_{n}\left(s_{1}\right)-u_{n}\left(s_{2}\right)\right\|+\quad \sup \quad\left\|u_{n}\left(s_{1}\right)-u_{n}\left(s_{2}\right)\right\| \\
& -r \leq s_{1}, s_{2} \leq 0 \quad-r \leq s_{1} \leq 0 \leq s_{2} \leq 1 \\
& \left|s_{1}-s_{2}\right| \leq \frac{1}{n} \quad\left|s_{1}-s_{2}\right| \leq \frac{1}{n} \\
& +\quad \sup \quad\left\|u_{n}\left(s_{1}\right)-u_{n}\left(s_{2}\right)\right\|+\left\|\tau(t) u_{n}-\tau(t) u\right\| \\
& 0 \leq s_{1} \leq s_{2} \leq 1 \\
& \left|s_{1}-s_{2}\right| \leq \frac{1}{n} \\
& \leq \sup _{-r \leq s_{1}, s_{2} \leq 0}\left\|\varphi\left(s_{1}\right)-\varphi\left(s_{2}\right)\right\|+\sup _{-r \leq s_{1} \leq 0}\left\|u_{n}\left(s_{1}\right)-u_{n}(0)\right\| \\
& \left|s_{1}-s_{2}\right| \leq \frac{1}{n} \quad\left|s_{1}\right| \leq \frac{1}{n} \\
& +\sup _{0 \leq s_{2} \leq 1}\left\|u_{n}(0)-u_{n}\left(s_{2}\right)\right\|+\sup _{0 \leq s_{1} \leq s_{2} \leq 1}\left\|u_{n}\left(s_{1}\right)-u_{n}\left(s_{2}\right)\right\| \\
& \left|s_{2}\right| \leq \frac{1}{n} \quad\left|s_{1}-s_{2}\right| \leq \frac{1}{n} \\
& +\left\|\tau(t) u_{n}-\tau(t) u\right\| \\
& \leq \quad 2 \sup \quad\left\|\varphi\left(s_{1}\right)-\varphi\left(s_{2}\right)\right\|+\quad 2 \sup \quad\left\|u_{n}\left(s_{1}\right)-u_{n}\left(s_{2}\right)\right\| \\
& -r \leq s_{1}, s_{2} \leq 0 \quad 0 \leq s_{1}, s_{2} \leq 1 \\
& \left|s_{1}-s_{2}\right| \leq \frac{1}{n} \quad\left|s_{1}-s_{2}\right| \leq \frac{1}{n} \\
& +\left\|\tau(t) u_{n}-\tau(t) u\right\| \text {. }
\end{aligned}
$$

By the continuity of $\varphi$, the uniform convergence of $u_{n}$ towards $u$ and the preceding estimate, we get

$$
\lim _{n \rightarrow \infty}\left\|\tau\left(\delta_{n}(t)\right) u_{n}-\tau(t) u\right\|=0
$$

Similarly, we can show that

$$
\lim _{n \rightarrow \infty}\left\|\tau\left(\delta_{n}(t)\right) w_{n}-\tau(t) w\right\|=0 .
$$


M.S. Abdo, A.G. Ibrahim, S.K. Panchal - Sweeping Process in Banach Spaces

Step 5. In this step we prove that the sequence $\left(f_{n}\right)$ defined by $f_{n}(t)=g_{n}\left(\delta_{n}(t)\right)$ converges pointwise to a function $f \in L^{1}\left(I, X^{*}\right)$ with $f(t) \in G(t, \tau(t) w, \tau(t) u)$, a.e. for $t \in I$.

Let $t \in I$ be fixed. In view of the properties $(i i),($ iii $)$ of (16) and condition $\left(G_{2}\right)$ we obtain, for $n \geq 1$

$$
\begin{aligned}
& \left\|f_{n+1}(t)-f_{n}(t)\right\| \\
= & \left\|g_{n+1}\left(\delta_{n+1}(t)\right)-g_{n}\left(\delta_{n}(t)\right)\right\| \\
\leq & d\left(g_{n}\left(\delta_{n}(t)\right), G\left(\delta_{n+1}(t), \tau\left(\delta_{n+1}\left(\delta_{n+1}(t)\right)\right) w_{n+1}, \tau\left(\delta_{n+1}\left(\delta_{n+1}(t)\right)\right) u_{n+1}\right)\right)+\frac{1}{(n+1)^{2}} \\
= & d\left(g_{n}\left(\delta_{n}(t)\right), G\left(\delta_{n+1}(t), \tau\left(\delta_{n+1}(t)\right) w_{n+1}, \tau\left(\delta_{n+1}(t)\right) u_{n+1}\right)\right)+\frac{1}{(n+1)^{2}} \\
\leq & d_{H}\left(G\left(\delta_{n}(t), \tau\left(\delta_{n}(t)\right) w_{n}, \tau\left(\delta_{n}(t)\right) u_{n}\right), G\left(\delta_{n+1}, \tau\left(\delta_{n+1}(t)\right) w_{n+1}, \tau\left(\delta_{n+1}(t)\right) u_{n+1}\right)\right) \\
& +\frac{1}{(n+1)^{2}} \\
\leq & \left|\Lambda\left(\delta_{n}(t)\right)-\Lambda\left(\delta_{n+1}(t)\right)\right|\left(\left\|\tau\left(\delta_{n}(t)\right) w_{n}-\tau\left(\delta_{n+1}(t)\right) w_{n+1}\right\|\right. \\
& \left.+\left\|\tau\left(\delta_{n}(t)\right) u_{n}-\tau\left(\delta_{n+1}(t)\right) u_{n+1}\right\|\right)+\frac{1}{(n+1)^{2}} \\
\leq & \left|\Lambda\left(\delta_{n}(t)\right)-\Lambda\left(\delta_{n+1}(t)\right)\right|(2\|\varphi(0)\|+\|\psi(0)\|+2 k)+\frac{1}{(n+1)^{2}} .
\end{aligned}
$$

Then for any two natural numbers $n, m(n<m)$ we get

$$
\begin{aligned}
& \left\|f_{n}(t)-f_{m}(t)\right\| \\
\leq & \left\|f_{n}(t)-f_{n+1}(t)\right\|+\left\|f_{n+1}(t)-f_{n+2}(t)\right\|+\ldots \ldots . .+\left\|f_{m-1}(t)-f_{m}(t)\right\| \\
\leq \quad & (2\|\varphi(0)\|+\|\psi(0)\|+2 k)\left[\left|\Lambda\left(\delta_{n}(t)\right)-\Lambda\left(\delta_{n+1}(t)\right)\right|\right. \\
& \left.+\left|\Lambda\left(\delta_{n+1}(t)\right)-\Lambda\left(\delta_{n+2}(t)\right)\right|+\ldots \ldots . .+\left|\Lambda\left(\delta_{m-1}(t)\right)-\Lambda\left(\delta_{m}(t)\right)\right|\right] \\
& +\frac{1}{(n+1)^{2}}+\frac{1}{(n+1)^{2}}+\ldots \ldots .+\frac{1}{m^{2}} \\
\leq & (2\|\varphi(0)\|+\|\psi(0)\|+2 k)\left|\Lambda\left(\delta_{n}(t)\right)-\Lambda\left(\delta_{m}(t)\right)\right|+\frac{m}{(n+1)^{2}} .
\end{aligned}
$$

From the fact that $\Lambda$ is continuous and $\lim _{n \rightarrow \infty} \delta_{n}(t)=\lim _{m \rightarrow \infty} \delta_{m}(t)=t$, the righthand side of (19) tends to zero when $n, m \rightarrow \infty$. Hence, $\left(f_{n}(t)\right)$ is a Cauchy sequence in $X^{*}$ and $\left(f_{n}\right)$ converges pointwise to a function $f \in L^{1}\left(I, X^{*}\right)$. Moreover, by $(i i)$, 
(iii) of (3) and Condition $\left(G_{2}\right)$ we get

$$
\begin{aligned}
& d(f(t), G(t, \tau(t) w, \tau(t) u)) \\
\leq & \left\|f(t)-f_{n}(t)\right\|+d\left(f_{n}(t), G(t, \tau(t) w, \tau(t) u)\right) \\
= & \left\|f(t)-f_{n}(t)\right\|+d\left(g_{n}\left(\delta_{n}(t)\right), G(t, \tau(t) w, \tau(t) u)\right) \\
\leq & \left\|f(t)-f_{n}(t)\right\|+d_{H}\left(G\left(\delta_{n}(t), \tau\left(\delta_{n}(t)\right) w_{n}, \tau\left(\delta_{n}(t)\right) u_{n}\right), G\left(t, \tau(t) w, \tau\left(\delta_{n}(t)\right) u\right)\right) \\
\leq & \left\|f(t)-f_{n}(t)\right\|+\left|\Lambda\left(\delta_{n}(t)\right)-\Lambda(t)\right|\left(\left\|\tau\left(\delta_{n}(t)\right) w_{n}-\tau(t) w\right\|+\left\|\tau\left(\delta_{n}(t)\right) u_{n}-\tau(t) u\right\|\right) .
\end{aligned}
$$

Again, by Step 4 and from the fact that $\Lambda$ is continuous, the right-hand side of this inequality tends to zero when $n \rightarrow \infty$. Hence, $f(t) \in G(t, \tau(t) w, \tau(t) u), t \in I$.

Step 6. We prove that $J\left(u^{\prime}(t)\right)-f(t) \in-N_{C(t, w(t))}\left(u^{\prime}(t)\right)$, for a.e. $t \in I$.

Since $X$ is uniformly smooth, the duality map $J$ is uniformly continuous on bounded set, then the sequence $J\left(u_{n}^{\prime}\right)-f_{n}$ converges weakly towards $J\left(u^{\prime}\right)-f$ in $L^{1}\left(I, X^{*}\right)$, where $J\left(u_{n}^{\prime}\right)(t)=J\left(u_{n}^{\prime}(t)\right)$ and $J\left(u^{\prime}\right)(t)=J\left(u^{\prime}(t)\right)$. It follows by the Mazur's Lemma

$$
J\left(u^{\prime}(t)\right)-f(t) \in \bigcap_{n} \overline{c o}\left\{J\left(u_{j}^{\prime}(t)\right)-f_{j}(t): j \geq n\right\} \text {, for a.e. } t \in I .
$$

Fix any $t$ such that the preceding relation is satisfied and consider $\zeta \in X$. The last relation above yields

$$
\left\langle J\left(u^{\prime}(t)\right)-f(t), \zeta\right\rangle \leq \inf _{n} \sup _{j \geq n}\left\langle J\left(u_{j}^{\prime}(t)\right)-f_{j}(t), \zeta\right\rangle .
$$

Moreover, the relations (viii), (ix) of (16) and definition of $f_{n}$ tell us for a.e. $t \in I$

$$
J\left(u_{n}^{\prime}(t)\right)-f_{n}(t) \in-N_{C\left(\theta_{n}(t), w_{n}\left(\theta_{n}(t)\right)\right)}\left(u_{n}^{\prime}(t)\right) \cap \mathbb{B}(0, k+\mu) .
$$

Hence, by Lemma 3, for a.e. $t \in I$ we have

$$
J\left(u_{n}^{\prime}(t)\right)-f_{n}(t) \in-(k+\mu) \partial d_{C\left(\theta_{n}(t), w_{n}\left(\theta_{n}(t)\right)\right)}\left(u_{n}^{\prime}(t)\right) .
$$

In view of (20) and (21) for $\zeta \in X$ and for a.e. $t \in I$ one obtains

$$
\begin{aligned}
& \left\langle J\left(u^{\prime}(t)\right)-f(t), \zeta\right\rangle \\
\leq & \lim _{n \rightarrow \infty} \sup \left\langle J\left(u_{n}^{\prime}(t)\right)-f_{n}(t), \zeta\right\rangle \\
\leq & (k+\mu) \lim _{n \rightarrow \infty} \sup \delta^{*}\left(\zeta,-\partial d_{C\left(\theta_{n}(t), w_{n}\left(\theta_{n}(t)\right)\right)}\left(u_{n}^{\prime}(t)\right)\right) .
\end{aligned}
$$

From the u.s.c property, Lemma 4 and the last relation above yield for $\zeta \in X$, a.e. $t \in I$

$$
\left\langle J\left(u^{\prime}(t)\right)-f(t), \zeta\right\rangle \leq(k+\mu) \delta^{*}\left(\zeta,-\partial d_{C(t, w(t))}\left(u^{\prime}(t)\right)\right) .
$$


M.S. Abdo, A.G. Ibrahim, S.K. Panchal - Sweeping Process in Banach Spaces

As the set $\partial d_{C(t, w(t))}\left(u^{\prime}(t)\right)$ is closed and convex and $u^{\prime}(t) \in C(t, w(t))$ we get

$$
J\left(u^{\prime}(t)\right)-f(t) \in-(k+\mu) \partial d_{C(t, w(t))}\left(u^{\prime}(t)\right), \text { for a.e. } t \in I .
$$

Therefore,

$$
J\left(u^{\prime}(t)\right)-f(t) \in-N_{C(t, w(t))}\left(u^{\prime}(t)\right) \text {, for a.e. } t \in I .
$$

Finally, by Steps 5 and 6 we have, for almost $t \in I$

$$
J\left(u^{\prime}(t)\right) \in-N_{C(t, w(t))}\left(u^{\prime}(t)\right)+G(t, \tau(t) w, \tau(t) u) .
$$

Which completes the proof.

Remark 1. we can prove the relation $u^{\prime}(t) \in C(t, w(t))$, for a.e. $t \in I$ as follows:

Since the sequence $\left(u_{n}^{\prime}\right)$ converges weakly to $u^{\prime}$ in $L^{1}(I, X)$, then invoking Remark 3.1 in [20], we get for a.e. $t \in I$,

$$
\begin{aligned}
u^{\prime}(t) & \in \overline{\operatorname{conv}}^{w}\left(\lim _{n \rightarrow \infty} \sup u_{n}^{\prime}(t)\right) \\
& \subseteq \overline{\operatorname{conv}}^{w}\left(\lim _{n \rightarrow \infty} \sup C\left(\theta_{n}(t), w_{n}\left(\theta_{n}(t)\right)\right)\right) .
\end{aligned}
$$

From the upper semicontinuity of $C$, we infer that $u^{\prime}(t) \in C(t, w(t))$, for a.e. $t \in I$.

\section{REFERENCES}

[1] R. P. Agarwal, D. O'Regan and D. R. Sahu, Fixed Point Theory for Lipschitzian-type Mappings with Applications, Springer, London, New York. 2009.

[2] F. A. Aladsani and A. G. Ibrahim, Non compact perturation of sweeping process with dealy in Banach spaces, International J. Math. and Math. Sciences. Article ID 567094. 2013, (2013), 1-10.

[3] Ya. I. Al'ber and I. Ryazantseva, Nonlinear III-Posed Problems of Monotone Type, Springer Dordrecht 2006.

[4] Ya. I. Al'ber, Generalized projection operators in Banach spaces: properties and applications, Functional Differ. Equ. 1, (1994), 1-21.

[5] M. Aitalioubrahim, On noncompact perturbation of nonconvex sweeping process, Comment. Math. Univ. Carolina, 53, 1 (2012), 65-77.

[6] J. P. Aubin and A. Cellina, Differential Inclusions, Set-Valued Maps and Viability Theory, Springer-Verlag, Berlin, 1984. 
M.S. Abdo, A.G. Ibrahim, S.K. Panchal - Sweeping Process in Banach Spaces

[7] M. Bounkhel and C. Castaing, State dependent sweeping process in p-uniformly smooth and q-uniformly convex Banach space, Set-Valued Var. Anal. 20, 2 (2012), 187-201.

[8] M. Bounkhel and R.Al-Yuosf, First and second order convex sweeping processes in reflexive smooth Banach spaces, Set-Valued Anal. 18, 2 (2010), 151-182.

[9] M. Bounkhel, Existence and uniqueness of some variants of non-convex sweeping process, J. Nonlinear Conv. Anal. 8, 2 (2007), 311-323.

[10] M. Bounkhel and L. Thibault, Non-Convex sweeping process and prox-regular in Hilbert spaces, J. Nonlinear Conv. Anal. 6, 2 (2005), 359-374.

[11] C. Castaing, A. G. Ibrahim and M. Yarou, Some contributions to nonconvex sweeping process, J. Nonlinear Conv. Anal. 10, 1 (2009), 1-20.

[12] C. Castaing and M. Valadier, Convex Analysis and Measurable Multifunctions, Lectures Notes in Mathematics, Springer-Verlag, New York, Berlin, 1977.

[13] F. H. Clarke, Optimization and Nonsmooth Analysis, John Wiley \& Sons Inc., New York, 1983.

[14] R. Deville, G. Godefroy and V. Zizler, Smoothness and Renormings in Branch Spaces, Pitman Monographs and Surveys in Pure and Applied Mathematics, Longman Scientific \& Technical, Harlow, UK, 1993.

[15] J. Diestel, Geometry of Banach Spaces, Selected Topics, Lecture Notes in Mathematics 485, Springer-Verlag, 1975.

[16] J. F. Edmond and L. Thibault, BV Solutions of non-convex sweeping process differential inclusions with perturbations, J. Diff. Equ. 226, 1 (2006), 133-179.

[17] A. G. Ibrahim and F. A. AL-Adsani, Convex sweeping processes with noncompact perturbations and with delay in Banach Spaces, Abstr. Appl. Anal. Hindawi Publishing Corporation. 2016, (2016).

[18] A. G. Ibrahim and F. A. AL-Adsani, Second order evolutions inclusions governed by sweeping process in Banach spaces, Le Matematiche. LXIV. (II. 64, 2 (2009), 17-39.

[19] J. J. Moreau, Evolution problem associated with a moving convex set in a Hilbert Space, J. Diff. Equ. 26, 3 (1977), 347-374.

[20] N. S. Papageorgiou, Convergence theorems for Banach space valued integrable multifunctions, Inter. J. of Math. Sci. 10, 3 (1987), 433-442.

[21] W. Takahashi, Nonlinear Functional Analysis, Yokohama, 2000.

[22] L. Thibault, Requalarization of non-convex sweeping process in Hilbert Spaces, Set-Valued Anal. 16, 2 (2008), 319-333.

[23] L. Thibault, Sweeping process with regular and nonregular sets, J. Diff. Equ. 193, 1 (2003), 1-26. 
M.S. Abdo, A.G. Ibrahim, S.K. Panchal - Sweeping Process in Banach Spaces

[24] Q. J. Zhu, On the solution set of differential inclusions in Banach space, J. Diff. Equ. 93, 2 (1991), 213-237.

Mohammed S. Abdo

${ }^{1}$ Research Scholar at Department of Mathematics, Dr. Babasaheb Ambedkar Marathwada University Aurangabad 431004 (M.S.), India

${ }^{2}$ Department of Mathematics, Hodeidah University, Al-Hodeidah 3114, Yemen

email: msabdo1977@gmail.com

Ahmed G. Ibrahim

Department of Mathematics, Factuly of Science, King Faisal University

Alhasa, Saudi Arabia

email: agamal2000@yahoo.com

Satish K. Panchal

Department of Mathematics, Dr. Babasaheb Ambedkar Marathwada University

Aurangabad, India

email: drpanchalsk@gmail.com 